Findings 6274 cases were identified from 73 million people. There was a linear relationship between $\log$ incidence and $\log$ age in all five registers: Ireland $\mathrm{R} 2=0.99$, Netherlands $\mathrm{R} 2=0.99$, Italy $\mathrm{R} 2=0.95$, Scotland $\mathrm{R} 2=0.97$, England $R 2=0 \cdot 95$, overall $R 2=0 \cdot 99$. All five registers gave very similar estimates of the linear slope ranging from $4 \cdot 5$ to $5 \cdot 1$, with overlapping confidence intervals. Combining all five registers gave an overall slope of 4.8 (95\% CI $4.5,5 \cdot 0)$ with similar estimates for males and females. In persons with known genetic mutations which predispose to ALS, the risk of ALS was higher, but the log-incidence/log-age slope was lower, as predicted by the multistage modelo.

Interpretation A linear relationship between the log incidence and log age of onset of ALS is consistent with a multistage model of disease. The slope estimate is about 5, suggesting that ALS is a 6-step process; the slope is 3-4 in persons with known genetic causes. These findings have implications for the search for causes of ALS, including occupational causes and gene-environment interactions.

\section{O6E.4 METABOLOME AND EXPOSOME PROFILING: NEW OPPORTUNITIES TO STUDY RISK FACTORS FOR PARKINSON'S DISEASE}

\begin{abstract}
${ }^{1,2}$ Susan Peters, ${ }^{1,3}$ Douglas Walker, ${ }^{4}$ Gary Miller, ${ }^{1,5}$ Marc Chadeau-Hyam, ${ }^{5}$ Paolo Vineis, ${ }^{6}$ Valentina Gallo, ${ }^{1,7}$ Roel Vermeulen*. ${ }^{1}$ Institute for Risk Assessment Sciences, Utrecht University, Utrecht, Netherlands; ${ }^{2}$ Neurology Department, University Medical Centre Utrecht, Utrecht, Netherlands; ${ }^{3}$ Icahn School of Medicine, Mount Sinai, USA; ${ }^{4}$ Emory University, Atlanta, USA; ${ }^{5}$ School of Public Health, Imperial College, London, UK; ${ }^{6}$ Queen Mary University of London, London, UK; ${ }^{7} J u l i u s$ Center, University Medical Centre Utrecht, Utrecht, Netherlands
\end{abstract}

\subsection{6/OEM-2019-EPI.161}

Parkinson's disease (PD) is the second most common neurodegenerative disorder after Alzheimer disease and is imposing an increasing social and economic burden in ageing populations. Although the role of environmental factors has been recognised, few established risk factors have been consistently identified. Evidence that exposure to pesticides, herbicides and metals increase PD risk is suggestive, but further research is needed to identify specific compounds that may play a causal role.

Large established prospective population studies offer an important opportunity for investigating risk factors in relatively rare diseases such as PD. Within the European Prospective Investigation into Cancer and Nutrition (EPIC) cohort, 734 incident PD cases have been ascertained, for whom prediagnostic blood has been stored. A nested case-control study will be conducted, where one control per case will be selected by incidence density sampling matched by age at recruitment, sex and study centre.

Untargeted metabolomics can simultaneously characterize thousands of endogenous and exogenous compounds within a biological sample: the metabolome. Metabolome wide association studies (MWAS) have identified significant differences in the metabolomic profile of older adults with and without PD. We will employ an innovative approach combining liquid and gas phase chromatography with ultra-highresolution mass spectrometry (LC/GC-UHRMS), to identify exogenous chemical exposures (e.g. pollutants, pesticides and medications), the exposome, in addition to the metabolome.
Disease specific variability in blood metabolite compositions may signify the presence of mechanistic aberrations contributing to PD pathogenesis. The combination of metabolome and exposome profiling provides a measure of the continuum from exposure to disease. Allowing previously unavailable richness and depth for characterizing the metabolome and exposome upon which novel discoveries in PD can be made.

The EPIC cohort allows us to perform a to study the metabolome and exposome well before disease onset, to eliminate possible effects of levodopa medication or disease-related processes.

\section{O6E.5 OCCUPATION AND RISK OF AMYOTROPHIC LATERALS SCLEROSIS (ALS) IN DENMARK} ${ }^{2,3}$ Aisha Dickerson, ${ }^{1}$ Johnni Hansen*, ${ }^{5}$ Marianthi-Anna Kiumourzoglou, ${ }^{3}$ Aron Specht,
${ }^{4}$ Ole Gredal, ${ }^{2,3}$ Marc Weisskopf. ${ }^{1}$ Danish Cancer Society Research Center, København,
Denmark; ${ }^{2}$ Department of Epdemilogy, Harvard T.H. Chan School of Public Health, Boston,
USA; ${ }^{3}$ Department of Environmental Health, Harvard T.H. Chan School of Public Health,
Boston, USA; ${ }^{4}$ Danish Rehabilitation Center, Copenhagen, Denmark; ${ }^{5}$ Department of
Environmental Health Services, Columbia University Mailman School of Public Health, New
York, USA

\subsection{6/OEM-2019-EPI.162}

Introduction Amyotrophic lateral sclerosis (ALS) is a rare and complex neurodegenerative disease, which is highly fatal. It is known that $5 \%-10 \%$ of ALS cases are hereditary, but apart from this the causes for sporadic of ALS are unknown. Some evidence, however, suggests that manifestation and progression may be associated with certain occupational exposures, e.g. exposure to formaldehyde, lead and military service. The aim of this study is to survey the occupational risk of ALS in Denmark based on nationwide registries.

Methods We identified 1826 ALS cases who were 25 years old or less in 1964 and diagnosed from 1982 to 2013 from the Danish National Patient Registry, which cover all hospitals in Denmark. We matched 100 ALS free population controls to each case based on birth year and sex. Information on demographic data were obtained from the national Population Register and linked by the unique personal indentifier, assigned to all residents, to the Danish Pension Fund (DPF) to determine individual employment history from 1964. All employees in Denmark are compulsory members of DPF. Conditional logistic regression models were uses for estimation of odds ratios OR and their 95\% confidence intervals, adjusted for socioeconomic status, marital status and residential location.

Results We observed increased OR of ALS among men who worked in e.g. agriculture, hunting, forestry or fishing $(\mathrm{OR}=1.2 ; 1.0-1.5)$. There was also a positive association for men employed in construction $(\mathrm{OR}=1.2 ; 1.1-1.4)$. In women, no significant increases were observed, but a protective association was seen with employment in the cleaning industry $(\mathrm{OR}=0.7 ; 0.5-0.9)$.

Conclusions Our study shows various occupations with exposure to toxicants, such as diesel exhaust and lead, and strenuous physical activity associated with increased odds of ALS in men. Future studies should have a particular focus on gathering detailed information on physical exertion and exposure to specific chemicals. 